\title{
An entanglement criterion for states in infinite-dimensional multipartite quantum systems
}

\author{
WANG YinZhu ${ }^{1,2}$, HOU JinChuan ${ }^{1 *} \&$ GUO Yu ${ }^{1,3}$ \\ ${ }^{1}$ School of Mathematics, Taiyuan University of Technology, Taiyuan 030024, China; \\ ${ }^{2}$ Department of Mathematics, Taiyuan University of Science and Technology, Taiyuan 030024, China; \\ ${ }^{3}$ Department of Mathematics, Shanxi Datong University, Datong 037009, China
}

Received October 17, 2011; accepted December 14, 2011

\begin{abstract}
In (Phys Lett A, 2002, 297: 4-8) an entanglement criterion for finite-dimensional bipartite systems is proposed: If $\rho_{A B}$ is a separable state, then $\operatorname{Tr}\left(\rho_{A}^{2}\right) \geqslant \operatorname{Tr}\left(\rho^{2}\right)$ and $\operatorname{Tr}\left(\rho_{B}^{2}\right) \geqslant \operatorname{Tr}\left(\rho^{2}\right)$. In the present paper this criterion is extended to infinite-dimensional bipartite and multipartite systems. The reduction criterion presented in (Phys Rev A, 1999, 59: 4206-4216) is also generalized to infinitedimensional case. Then it is shown that the former criterion is weaker than the later one.
\end{abstract}

quantum state, entanglement criterion, infinite-dimensional system

Citation: Wang Y Z, Hou J C, Guo Y. An entanglement criterion for states in infinite-dimensional multipartite quantum systems. Chin Sci Bull, 2012, 57: 1643-1647, doi: 10.1007/s11434-012-5111-5

\section{Introduction}

Quantum entanglement plays a crucial role in the rapidly developing theory of quantum information and quantum computation [1]. One of the most fundamental problem in entanglement theory is to determine whether a given quantum state is entangled or not. Nowadays, a number of different entanglement criteria have been found [2-18].

For the finite-dimensional bipartite quantum systems, $\mathrm{Wu}$ and Anandan [18] proposed a trace inequality criterion which reads as: If $\rho$ is a separable state of a bipartite quantum system $A+B$, then $\operatorname{Tr}\left(\rho_{A}^{2}\right) \geqslant \operatorname{Tr}\left(\rho^{2}\right)$ and $\operatorname{Tr}\left(\rho_{B}^{2}\right) \geqslant \operatorname{Tr}\left(\rho^{2}\right)$, where $\rho_{A}$ and $\rho_{B}$ are the reduced density matrices of $\rho$, i.e. $\rho_{A}=\operatorname{Tr}_{B}(\rho)$ and $\rho_{B}=\operatorname{Tr}_{A}(\rho)$. Recall that, the reduction criterion says that if a state $\rho$ is separable, then $\rho_{A} \otimes I_{B}-\rho \geqslant 0$ and $I_{A} \otimes \rho_{B}-\rho \geqslant 0$. It is claimed in [19] that the trace inequality criterion mentioned above is weaker than the reduction criterion. Though this claim is true, the proof of it is not right there.

The aim of this paper is to generalize the above two criteria to infinite-dimensional case and then show that the trace

*Corresponding author (email: jinchuanhou@yahoo.com) inequality criterion is weaker than the reduction criterion.

Recall that, a quantum state $\rho$ (i.e. a positive trace-one operator) acting on a Hilbert space $H=H_{A} \otimes H_{B}$ with $\operatorname{dim} H_{A} \otimes H_{B} \leqslant+\infty$, is called separable if it can be written as a convex combination of some product states, i.e. $\rho$ is of the form

$$
\rho=\sum_{i} p_{i} \rho_{i}^{A} \otimes \rho_{i}^{B}, \quad \sum_{i} p_{i}=1, p_{i} \geqslant 0
$$

or, it can be approximated in the trace norm by the states of the form in eq. (1) [20], where $\rho_{i}^{A}$ and $\rho_{i}^{B}$ are (pure) states in the subsystems $H_{A}$ and $H_{B}$, respectively. Otherwise, $\rho$ is called entangled. A quantum state $\rho$ (i.e. a positive trace-one operator) acting on a Hilbert space $H=H_{1} \otimes H_{2} \otimes \ldots \otimes H_{m}$ with $\operatorname{dim} H_{1} \otimes H_{2} \otimes \ldots \otimes H_{m} \leqslant+\infty$ is defined to be fully separable if it has the form

$$
\rho=\sum_{i} p_{i} \rho_{i}^{(1)} \otimes \rho_{i}^{(2)} \otimes \ldots \otimes \rho_{i}^{(m)}, \quad \sum_{i} p_{i}=1, p_{i} \geqslant 0,
$$

or it can be approximated in the trace norm by the states of the above form, where $\rho_{i}^{(k)} \mathrm{s}$ are (pure) states in the subsystems $H_{k}, k=1,2, \ldots, m$. 
We fix some notations used in this paper. In quantum mechanics, a quantum system is associated with a separable complex Hilbert space $H$, i.e. the state space. Let $H_{A}, H_{B}$, etc., be complex separable Hilbert spaces (associated with quantum systems), throughout the paper we use the Dirac's symbols. The brackets notation, $\langle\cdot \mid \cdot\rangle$ stand for the inner product in the given Hilbert spaces. The set of all bounded linear operators on some Hilbert space $H$ is denoted by $B(H)$. Let $S\left(H_{A} \otimes H_{B}\right)$ be the set of all states in $H_{A} \otimes H_{B}$. It is obvious that $S\left(H_{A} \otimes H_{B}\right) \subseteq B\left(H_{A} \otimes H_{B}\right)$. By $\mathcal{T}(H)$ we denote the set of all trace class operators of Hilbert spaces $H$. If $T \in \mathcal{T}(H)$, we have $\|T\|_{T r}=\operatorname{Tr}\left(\left(T^{\dagger} T\right)^{\frac{1}{2}}\right)<+\infty$, where $\|\cdot\|_{T r}$ denote the trace norm. Recall that, if a quantum system is in one of a number of states $\left|\psi_{i}\right\rangle$, where $i$ is an index, with respective probabilities $p_{i}$, then $\left\{p_{i},\left|\psi_{i}\right\rangle\right\}$ is called an ensemble of pure states, and the associated density operator for the system is defined by $\rho=\sum_{i} p_{i}\left|\psi_{i}\right\rangle\left\langle\psi_{i}\right|$. Obviously, $\rho$ is a pure state if and only if $\operatorname{Tr}\left(\rho^{2}\right)=1$.

\section{The main results}

In this section we present the main results and their proofs.

Our first result is an infinite-dimensional version of the trace inequality criterion proposed in [18].

Theorem 2.1 Let $H_{A}, H_{B}$ be complex separable Hilbert spaces with $\operatorname{dim}\left(H_{A} \otimes H_{B}\right)=+\infty$. If $\rho \in S\left(H_{A} \otimes H_{B}\right)$ is separable, then $\operatorname{Tr}\left(\rho_{A}^{2}\right) \geqslant \operatorname{Tr}\left(\rho^{2}\right)$ and $\operatorname{Tr}\left(\rho_{B}^{2}\right) \geqslant \operatorname{Tr}\left(\rho^{2}\right)$, where $\rho_{A}=\operatorname{Tr}_{B}(\rho), \rho_{B}=\operatorname{Tr}_{A}(\rho)$.

In order to prove this theorem, we need a lemma.

Lemma 2.2 Let $H$ be a complex Hilbert space, $T_{n}, T \in$ $\mathcal{T}(H)$. Then $\lim _{n \rightarrow+\infty} T_{n}=T$ under the trace norm implies that $\lim _{n \rightarrow+\infty} T_{n}^{2}=T^{2}$ under the trace norm.

Proof. $\quad$ Note that, for $A \in \mathcal{T}(H)$, we have $\|A\| \leqslant\|A\|_{\operatorname{Tr}}<$ $+\infty$. As $T_{n}, T \in \mathcal{T}(H) \subseteq B(H)$ and $\left\|T_{n}-T\right\|_{T r} \rightarrow 0$, there exists some positive number $M$ such that $\sup _{n}\left\{\left\|T_{n}\right\|_{\mathrm{Tr}}\right\}=M<$ $+\infty$. Then, it follows from

$$
\begin{aligned}
& \left\|T_{n}^{2}-T^{2}\right\|_{\operatorname{Tr}} \\
= & \left\|T_{n}^{2}-T_{n} T+T_{n} T-T^{2}\right\|_{T r} \\
\leqslant & M \cdot\left\|T_{n}-T\right\|_{T r}+\|T\| \cdot\left\|T_{n}-T\right\|_{T r}
\end{aligned}
$$

that

$$
\left\|T_{n}^{2}-T^{2}\right\|_{\operatorname{Tr}} \rightarrow 0 \text { as } n \rightarrow+\infty .
$$

Proof of Theorem 2.1. Let $S_{S-p}\left(H_{A} \otimes H_{B}\right)$ be the set of all separable pure states. If $\rho$ is separable, then it admits a representation of Bochner integral [21]

$$
\rho=\int_{S_{S-p}} \varphi\left(\rho^{A} \otimes \rho^{B}\right) \mathrm{d} \mu\left(\rho^{A} \otimes \rho^{B}\right),
$$

where $\mu$ is a Borel probability measure on $S_{S-p}\left(H_{A} \otimes H_{B}\right)$, $\rho^{A} \otimes \rho^{B} \in S_{S-p}\left(H_{A} \otimes H_{B}\right)$, and $\varphi: S_{S-p} \rightarrow S_{S-p}$ is a measurable function. It follows that there exists a sequences of step function $\varphi_{n}$, such that

$$
\varphi\left(\rho^{A} \otimes \rho^{B}\right)=\lim _{n \rightarrow+\infty} \varphi_{n}\left(\rho^{A} \otimes \rho^{B}\right)
$$

with respect to the trace norm, where

$$
\varphi_{n}\left(\rho^{A} \otimes \rho^{B}\right)=\sum_{i=1}^{k_{n}} \mathcal{X}_{E_{i}}\left(\rho^{A} \otimes \rho^{B}\right) \rho_{i}^{A} \otimes \rho_{i}^{B},
$$

and $\mathcal{X}_{E_{i}}(\cdot)$ is the characteristic function of $E_{i},\left\{E_{i}\right\}_{i=1}^{k_{n}}$ is a partition of $S_{S-p}\left(H_{A} \otimes H_{B}\right)$. Denote by $E$ the set of all possible partitions $\left\{E_{i}\right\}_{i=1}^{k_{n}}$ of $S_{S-p}\left(H_{A} \otimes H_{B}\right)$. Then $E$ is a direct set and we have

$$
\rho=\lim _{\left\{E_{i}\right\} \in E} \sum_{i} \mu\left(E_{i}\right) \rho_{i}^{A} \otimes \rho_{i}^{B},
$$

with respect to the trace norm, as well as with respect to the Hilbert Schmidt norm, where $\rho_{i}^{A}$ and $\rho_{i}^{B}$ are pure states respectively in $H_{A}$ and $H_{B}$, i.e. there exist unit vectors $\left\{\left|\psi_{i}\right\rangle\right\}$ in $H_{A}$ and $\left\{\left|\phi_{i}\right\rangle\right\}$ in $H_{B}$, respectively, such that $\rho_{i}^{A}=\left|\psi_{i}\right\rangle\left\langle\psi_{i}\right|$ and $\rho_{i}^{B}=\left|\phi_{i}\right\rangle\left\langle\phi_{i}\right|$. It is well known that $\operatorname{Tr}$ is a completely positive linear functional of $\mathcal{T}(H)$ with $\operatorname{dim} H \leqslant+\infty$. In fact, taking any orthonormal basis $\left\{\left|e_{i}\right\rangle\right\}_{i=1}^{\operatorname{dim} H}$ of $H$, the complete positivity of $\operatorname{Tr}$ comes from the fact $\operatorname{Tr}(T)\left(\left|e_{1}\right\rangle\left\langle e_{1}\right|\right)=\sum_{i=1}^{\infty} E_{i} T E_{i}^{\dagger}$, where $E_{i}=\left|e_{1}\right\rangle\left\langle e_{i}\right|$. Thus $\operatorname{Tr}$ is completely bounded and hence $I \otimes \operatorname{Tr}$ is continuous. So we have

$$
\rho_{A}=\operatorname{Tr}_{B}(\rho)=\lim _{\left\{E_{i}\right\} \in E} \sum_{i} \mu\left(E_{i}\right) \rho_{i}^{A},
$$

with respect to the trace norm. By Lemma 2.2, one gets

$$
\begin{aligned}
\operatorname{Tr}\left(\rho_{A}^{2}\right) & =\lim _{\left\{E_{i}\right\} \in E} \operatorname{Tr}\left(\left[\sum_{i} \mu\left(E_{i}\right) \rho_{i}^{A}\right]^{2}\right) \\
& =\lim _{\left\{E_{i}\right\} \in E} \operatorname{Tr}\left(\sum_{i} \sum_{j} \mu\left(E_{i}\right) \mu\left(E_{j}\right) \rho_{i}^{A} \rho_{j}^{A}\right) \\
& =\lim _{\left\{E_{i}\right\} \in E} \operatorname{Tr}\left(\sum_{i} \sum_{j} \mu\left(E_{i}\right) \mu\left(E_{j}\right)\left|\psi_{i}^{A}\right\rangle\left\langle\psi_{i}^{A}|\cdot| \psi_{j}^{A}\right\rangle\left\langle\psi_{j}^{A}\right|\right) \\
& =\lim _{\left\{E_{i}\right\} \in E}\left[\sum_{i} \sum_{j} \mu\left(E_{i}\right) \mu\left(E_{j}\right)\left|\left\langle\psi_{i}^{A} \mid \psi_{j}^{A}\right\rangle\right|^{2}\right] \\
& \geqslant \lim _{\left\{E_{i}\right\} \in E}\left[\sum_{i} \sum_{j} \mu\left(E_{i}\right) \mu\left(E_{j}\right)\left|\left\langle\psi_{i}^{A} \mid \psi_{j}^{A}\right\rangle\left\langle\phi_{i}^{B} \mid \phi_{j}^{B}\right\rangle\right|^{2}\right] .
\end{aligned}
$$

On the other hand, by Lemma 2.2, we have

$$
\begin{aligned}
\operatorname{Tr}\left(\rho^{2}\right) & =\lim _{\left\{E_{i}\right\} \in E} \operatorname{Tr}\left(\left[\sum_{i} \mu\left(E_{i}\right) \rho_{i}^{A} \otimes \rho_{i}^{B}\right]^{2}\right) \\
& =\lim _{\left\{E_{i}\right\} \in E} \operatorname{Tr}\left(\sum_{i} \sum_{j} \mu\left(E_{i}\right) \mu\left(E_{j}\right) \rho_{i}^{A} \otimes \rho_{i}^{B} \rho_{j}^{A} \otimes \rho_{j}^{B}\right) \\
& =\lim _{\left\{E_{i}\right\} \in E}\left[\sum_{i} \sum_{j} \mu\left(E_{i}\right) \mu\left(E_{j}\right)\left|\left\langle\psi_{i}^{A} \mid \psi_{j}^{A}\right\rangle\left\langle\phi_{i}^{B} \mid \phi_{j}^{B}\right\rangle\right|^{2}\right] .
\end{aligned}
$$

So we obtain that $\operatorname{Tr}\left(\rho_{A}^{2}\right) \geqslant \operatorname{Tr}\left(\rho^{2}\right)$. The inequality $\operatorname{Tr}\left(\rho_{B}^{2}\right) \geqslant$ $\operatorname{Tr}\left(\rho^{2}\right)$ can be checked similarly. This completes the proof.

We give an example to illustrate how to apply the trace inequality criterion established in Theorem 2.1. 
Example Let $H=H_{A} \otimes H_{B}$ be a Hilbert space with $\operatorname{dim} H_{A}=2$ and $\operatorname{dim} H_{B}=+\infty$. We consider a state $\rho \in S\left(H_{A} \otimes H_{B}\right)$ with the following form:

$$
\begin{aligned}
\rho= & \frac{x}{2}\left|00^{\prime}\right\rangle\left\langle0 0 ^ { \prime } \left|+\frac{1-x}{2}\left(\left|01^{\prime}\right\rangle+\left|10^{\prime}\right\rangle\right)\left(\left\langle 01^{\prime}\right|+\left\langle 10^{\prime}\right|\right)\right.\right. \\
& +\frac{x}{2}\left(\left|02^{\prime}\right\rangle+\left|11^{\prime}\right\rangle\right)\left(\left\langle 02^{\prime}\right|+\left\langle 11^{\prime}\right|\right),
\end{aligned}
$$

where $0 \leqslant x \leqslant 1,\{|0\rangle,|1\rangle\}$ is an orthonormal basis of $H_{A}$ and $\left\{\left|0^{\prime}\right\rangle,\left|1^{\prime}\right\rangle, \cdots\right\}$ is an orthonormal basis of $H_{B}$. Then

$$
\rho=\left(\begin{array}{cccccc|cccccc}
\frac{x}{2} & 0 & 0 & \ldots & 0 & \ldots & 0 & 0 & 0 & \ldots & 0 & \ldots \\
0 & \frac{1-x}{2} & 0 & \ldots & 0 & \ldots & \frac{1-x}{2} & 0 & 0 & \ldots & 0 & \ldots \\
0 & 0 & \frac{x}{2} & \ldots & 0 & \ldots & 0 & \frac{x}{2} & 0 & \ldots & 0 & \ldots \\
\vdots & \vdots & \vdots & \ddots & \vdots & \ldots & \vdots & \vdots & \vdots & \ddots & \vdots & \ldots \\
0 & 0 & 0 & \ldots & 0 & \ldots & 0 & 0 & 0 & \ldots & 0 & \ldots \\
\vdots & \vdots & \vdots & \ddots & \vdots & \ddots & \vdots & \vdots & \vdots & \ddots & \vdots & \ddots \\
\hline 0 & \frac{1-x}{2} & 0 & \ldots & 0 & \ldots & \frac{1-x}{2} & 0 & 0 & \ldots & 0 & \ldots \\
0 & 0 & \frac{x}{2} & \ldots & 0 & \ldots & 0 & \frac{x}{2} & 0 & \ldots & 0 & \ldots \\
0 & 0 & 0 & \ldots & 0 & \ldots & 0 & 0 & 0 & \ldots & 0 & \ldots \\
\vdots & \vdots & \vdots & \ddots & \vdots & \ldots & \vdots & \vdots & \vdots & \ddots & \vdots & \ldots \\
0 & 0 & 0 & \ldots & 0 & \ldots & 0 & 0 & 0 & \ldots & 0 & \ldots \\
\vdots & \vdots & \vdots & \ddots & \vdots & \ddots & \vdots & \vdots & \vdots & \ddots & \vdots & \ddots
\end{array}\right)
$$

and $\rho_{A}=\left(\begin{array}{cc}\frac{1+x}{2} & 0 \\ 0 & \frac{1}{2}\end{array}\right)$. It is obvious that

$$
\operatorname{Tr}\left(\rho^{2}\right)=\frac{9}{4} x^{2}-2 x+1 \quad \text { and } \quad \operatorname{Tr}\left(\rho_{A}^{2}\right)=\frac{1}{4}\left(x^{2}+2 x+2\right) \text {. }
$$

Thus $\operatorname{Tr}\left(\rho_{A}^{2}\right)<\operatorname{Tr}\left(\rho^{2}\right)$ whenever $0 \leqslant x<\frac{1}{4}$. So $\rho$ is entangled whenever $0 \leqslant x<\frac{1}{4}$.

For finite-dimensional case, the trace inequality criterion in [18] is valid for multipartite systems. Namely, if $\rho$ is a fully separable state acting on $H_{1} \otimes H_{2} \otimes \cdots \otimes H_{m}$, then

$$
\operatorname{Tr}\left(\rho_{\alpha_{1}}^{2}\right) \geqslant \operatorname{Tr}\left(\rho_{\alpha_{1} \alpha_{2}}^{2}\right) \geqslant \cdots \geqslant \operatorname{Tr}\left(\rho_{\alpha_{1} \alpha_{2} \cdots \alpha_{r}}^{2}\right) \geqslant \cdots \geqslant \operatorname{Tr}\left(\rho^{2}\right),
$$

where $\alpha_{1} \alpha_{2} \cdots \alpha_{r}$ represent $r$ distinct elements from the set $\{1,2, \cdots, m\}$, and $\rho_{\alpha_{1}}$ is the reduced density matrix of the subsystem $\alpha_{1}, \rho_{\alpha_{1} \alpha_{2}}$ is the reduced density operator of the system $\alpha_{1}+\alpha_{2}$, etc., that is, $\rho_{\alpha_{1}}$ is obtained by tracing over all subsystems except $\alpha_{1}, \rho_{\alpha_{1} \alpha_{2}}$ is obtained by tracing over all subsystems except $\alpha_{1}$ and $\alpha_{2}$, and so on. In what follows, we will generalize Theorem 2.1 to multipartite cases.

Let $H_{1}, H_{2}, \ldots, H_{m}$ be separable complex Hilbert spaces with $\operatorname{dim}\left(H_{1} \otimes H_{2} \otimes \cdots \otimes H_{m}\right)=+\infty$. By $S_{S-p}\left(H_{1} \otimes H_{2} \otimes \cdots \otimes H_{m}\right)$ we denote the set of all full separable pure states acting on $H_{1} \otimes H_{2} \otimes \cdots \otimes H_{m}$. According to [21], we have that if $\rho \in S\left(H_{1} \otimes H_{2} \otimes \cdots \otimes H_{m}\right)$ is fully separable, then $\rho$ admits a representation of Bochner integral

$$
\rho=\int_{S_{S-p}} \varphi\left(\rho^{(1)} \otimes \rho^{(2)} \otimes \cdots \otimes \rho^{(m)}\right) d \mu\left(\rho^{(1)} \otimes \rho^{(2)} \otimes \cdots \otimes \rho^{(m)}\right),
$$

where $\mu$ is a Borel probability measure on $S_{S-p}\left(H_{1} \otimes H_{2} \otimes\right.$ $\left.\cdots \otimes H_{m}\right), \rho^{(1)} \otimes \rho^{(2)} \otimes \cdots \otimes \rho^{(m)} \in S_{S-p}\left(H_{1} \otimes H_{2} \otimes \cdots \otimes H_{m}\right)$, and $\varphi: S_{S-p} \rightarrow S_{S-p}$ is a measurable function. It follows that there exists a sequences of step function $\varphi_{n}$ such that

$$
\varphi\left(\rho^{(1)} \otimes \rho^{(2)} \otimes \cdots \otimes \rho^{(m)}\right)=\lim _{n \rightarrow \infty} \varphi_{n}\left(\rho^{(1)} \otimes \rho^{(2)} \otimes \cdots \otimes \rho^{(m)}\right)
$$

with respect to the trace norm, where

$$
\begin{aligned}
\varphi_{n}\left(\rho^{(1)} \otimes \rho^{(2)} \otimes \cdots \otimes \rho^{(m)}\right)= & \sum_{i=1}^{k_{n}} \mathcal{X}_{E_{i}}\left(\rho^{(1)} \otimes \rho^{(2)} \otimes \cdots \otimes \rho^{(m)}\right) \\
& \times \rho_{i}^{(1)} \otimes \rho_{i}^{(2)} \otimes \cdots \otimes \rho_{i}^{(m)},
\end{aligned}
$$

$\mathcal{X}_{E_{i}}(\cdot)$ is the characteristic function of $E_{i}$, and $\left\{E_{i}\right\}_{i=1}^{k_{n}}$ is a partition of $S_{S-p}\left(H_{1} \otimes H_{2} \otimes \cdots \otimes H_{m}\right)$. Let us denote by $E$ the set of all finite partitions $\left\{E_{i}\right\}_{i=1}^{k_{n}}$ of $S_{S-p}\left(H_{1} \otimes H_{2} \otimes \cdots \otimes H_{m}\right)$. Now we have

$$
\rho=\lim _{\left\{E_{i}\right\} \in E} \sum_{i} \mu\left(E_{i}\right) \rho_{i}^{(1)} \otimes \rho_{i}^{(2)} \otimes \cdots \otimes \rho_{i}^{(m)}
$$

with respect to the trace norm, as well as with respect to the Hilbert Schmidt norm, where $\rho_{i}^{(j)}=\left|\psi_{i}^{j}\right\rangle\left\langle\psi_{i}^{j}\right|$ are pure states in $H_{j}, j=1,2, \ldots, m$.

Theorem 2.3 Let $H_{1}, H_{2}, \cdots, H_{m}$ be separable complex Hilbert spaces with $\operatorname{dim} H_{1} \otimes H_{2} \otimes \cdots \otimes H_{m}=+\infty$. If $\rho \in S\left(H_{1} \otimes H_{2} \otimes \cdots \otimes H_{m}\right)$ is a fully separable state, then

$$
\operatorname{Tr}\left(\rho_{\alpha_{1}}^{2}\right) \geqslant \operatorname{Tr}\left(\rho_{\alpha_{1} \alpha_{2}}^{2}\right) \geqslant \cdots \geqslant \operatorname{Tr}\left(\rho_{\alpha_{1} \alpha_{2} \cdots \alpha_{r}}^{2}\right) \geqslant \cdots \geqslant \operatorname{Tr}\left(\rho^{2}\right),
$$

where $\alpha_{1} \alpha_{2} \cdots \alpha_{r}$ represent $r$ distinct elements from the set $\{1,2, \ldots, m\}$, and $\rho_{\alpha_{1}}$ is the reduced density operator of the system $\alpha_{1}, \rho_{\alpha_{1} \alpha_{2}}$ is the reduced density operator of the system $\alpha_{1}+\alpha_{2}$, etc., that is, $\rho_{\alpha_{1}}$ is obtained by tracing over all subsystems except $\alpha_{1}, \rho_{\alpha_{1} \alpha_{2}}$ is obtained by tracing over all subsystems except $\alpha_{1}$ and $\alpha_{2}$, and so on.

Proof. Since $\operatorname{Tr}$ is a completely bounded functional, $\rho_{\alpha_{1} \alpha_{2} \cdots \alpha_{r}}$ is well-defined. From eq. (8), we can see that for every integer $r$ satisfying $1 \leqslant r \leqslant m, \rho_{\alpha_{1} \alpha_{2} \cdots \alpha_{r}}$ has a representation as follows:

$$
\rho_{\alpha_{1} \alpha_{2} \cdots \alpha_{r}}=\lim _{\left\{E_{i}\right\} \in E} \sum_{i} \mu\left(E_{i}\right) \rho_{i}^{\left(\alpha_{1}\right)} \otimes \rho_{i}^{\left(\alpha_{2}\right)} \otimes \cdots \otimes \rho_{i}^{\left(\alpha_{r}\right)} .
$$

By Lemma 2.2, we get

$$
\begin{aligned}
& \operatorname{Tr}\left(\rho_{\alpha_{1} \alpha_{2} \cdots \alpha_{r}}^{2}\right) \\
= & \lim _{\left\{E_{i}\right\} \in E} \operatorname{Tr}\left(\sum_{i, j} \mu\left(E_{i}\right) \mu\left(E_{j}\right)\left(\rho_{i}^{\left(\alpha_{1}\right)} \otimes \rho_{i}^{\left(\alpha_{2}\right)} \otimes \cdots \otimes \rho_{i}^{\left(\alpha_{r}\right)}\right)\right. \\
& \left.\times\left(\rho_{j}^{\left(\alpha_{1}\right)} \otimes \rho_{j}^{\left(\alpha_{2}\right)} \otimes \cdots \otimes \rho_{j}^{\left(\alpha_{r}\right)}\right)\right) \\
= & \lim _{\left\{E_{i}\right\} \in E}\left[\sum_{i} \sum_{j} \mu\left(E_{i}\right) \mu\left(E_{j}\right)\left|\left\langle\psi_{i}^{\alpha_{1}} \psi_{i}^{\alpha_{2}} \cdots \psi_{i}^{\alpha_{r}} \mid \psi_{j}^{\alpha_{1}} \psi_{j}^{\alpha_{2}} \cdots \psi_{j}^{\alpha_{r}}\right\rangle\right|^{2}\right]
\end{aligned}
$$




$$
\begin{aligned}
\geqslant & \lim _{\left\{E_{i}\right\} \in E}\left[\sum_{i} \sum_{j} \mu\left(E_{i}\right) \mu\left(E_{j}\right) \mid\left\langle\psi_{i}^{\alpha_{1}} \psi_{i}^{\alpha_{2}} \cdots \psi_{i}^{\alpha_{r}} \psi_{i}^{\alpha_{r+1}}\right|\right. \\
& \left.\left.\psi_{j}^{\alpha_{1}} \psi_{j}^{\alpha_{2}} \cdots \psi_{j}^{\alpha_{r}} \psi_{j}^{\alpha_{r+1}}\right\rangle\left.\right|^{2}\right] \\
= & \operatorname{Tr}\left(\rho_{\alpha_{1} \alpha_{2} \cdots \alpha_{r+1}}^{2}\right) .
\end{aligned}
$$

So we have

$$
\operatorname{Tr}\left(\rho_{\alpha_{1}}^{2}\right) \geqslant \operatorname{Tr}\left(\rho_{\alpha_{1} \alpha_{2}}^{2}\right) \geqslant \cdots \geqslant \operatorname{Tr}\left(\rho_{\alpha_{1} \alpha_{2} \cdots \alpha_{r}}^{2}\right) \geqslant \cdots \geqslant \operatorname{Tr}\left(\rho^{2}\right) .
$$

Next, we extend the reduction criterion to infinitedimensional case.

Theorem 2.4 Let $\rho \in S\left(H_{A} \otimes H_{B}\right)$ with $\operatorname{dim}\left(H_{A} \otimes H_{B}\right)=$ $+\infty$. If $\rho$ is a separable state, then

$$
\rho_{A} \otimes I_{B}-\rho \geqslant 0, \quad I_{A} \otimes \rho_{B}-\rho \geqslant 0 .
$$

Proof. We only need to prove the inequality $\rho_{A} \otimes I_{B}-\rho \geqslant 0$. Another one can be checked similarly. Since $\rho$ is separable, by eqs. (4) and (5), we have

$$
\rho=\lim _{\left\{E_{i}\right\} \in E} \sum_{i} \mu\left(E_{i}\right) \rho_{i}^{A} \otimes \rho_{i}^{B},
$$

and

$$
\rho_{A}=\operatorname{Tr}_{B}(\rho)=\lim _{\left\{E_{i}\right\} \in E} \sum_{i} \mu\left(E_{i}\right) \rho_{i}^{A}
$$

with respect to the trace norm. Then

$$
\begin{aligned}
& \rho_{A} \otimes I_{B}-\rho \\
= & \lim _{\left\{E_{i}\right\} \in E}\left(\sum_{i} \mu\left(E_{i}\right) \rho_{i}^{A} \otimes I_{B}-\sum_{i} \mu\left(E_{i}\right) \rho_{i}^{A} \otimes \rho_{i}^{B}\right) \\
= & \lim _{\left\{E_{i}\right\} \in E}\left[\sum_{i} \mu\left(E_{i}\right)\left(\rho_{i}^{A} \otimes I_{B}-\rho_{i}^{A} \otimes \rho_{i}^{B}\right)\right] \\
= & \lim _{\left\{E_{i}\right\} \in E}\left[\sum_{i} \mu\left(E_{i}\right)\left(\rho_{i}^{A} \otimes\left(I_{B}-\rho_{i}^{B}\right)\right)\right] \\
\geqslant & 0
\end{aligned}
$$

as desired.

Now we discuss the relation between the trace inequality criterion and the reduction criterion.

Definition 2.5 Let $(a)$ and $(b)$ be two necessary separability criteria. By $\varepsilon(a)$ and $\varepsilon(b)$, we denote the set of entangled states detected by $(a)$ and $(b)$, respectively. We say that $(a)$ is weaker than $(b)$ if $\varepsilon(a) \subseteq \varepsilon(b)$. In this case we also say that $(b)$ is stronger than $(a)$. We say that $(a)$ and $(b)$ are equivalent if $\varepsilon(a)=\varepsilon(b)$. Finally we say that $(a)$ and $(b)$ are independent if $(a)$ is neither weaker nor stronger than $(b)$.

In [19], it is pointed out that the trace inequality criterion is weaker than the reduction criterion for the finite-dimensional systems. However the proof provided there is not correct since the argument is based on an incorrect equation (see eq. (3) in [19], it is stated there that " $\ldots \sqrt{\rho}=\left(\sqrt{\rho_{1}} \otimes I\right) R^{\dagger}=$ $R\left(\sqrt{\rho_{1}}\right) \otimes I$. Therefore, $\rho=R^{\dagger}\left(\sqrt{\rho_{1}} \otimes I\right) R$ and ...". This is not correct since $\rho=R\left(\sqrt{\rho_{1}} \otimes I\right) R^{\dagger} \neq R^{\dagger}\left(\sqrt{\rho_{1}} \otimes I\right) R$. The fact is true that the trace inequality criterion is weaker than the reduction criterion for systems of any dimension. We give a correct proof that is valid for both finite-dimensional and infinite-dimensional cases.

Theorem 2.6 The trace inequality criterion is weaker than the reduction criterion for both finite- and infinitedimensional bipartite systems.

Proof. Assume that $\operatorname{dim} H_{A} \otimes H_{B} \leqslant+\infty$. Let $\rho \in$ $S\left(H_{A} \otimes H_{B}\right)$ be any state satisfying $\rho_{A} \otimes I_{B}-\rho \geqslant 0$. Since the square root is an operator monotone function, we have $\sqrt{\rho_{A}} \otimes I_{B}-\sqrt{\rho} \geqslant 0$. By Douglas theorem [22], there exists a contractive operator $R$ such that $\sqrt{\rho}=\left(\sqrt{\rho_{A}} \otimes I_{B}\right) R=$ $R^{\dagger}\left(\sqrt{\rho_{A}} \otimes I_{B}\right)$. It follows that

$$
\rho=\left(\sqrt{\rho_{A}} \otimes I_{B}\right) R R^{\dagger}\left(\sqrt{\rho_{A}} \otimes I_{B}\right) .
$$

Thus,

$$
\rho_{A}=\operatorname{Tr}_{B}(\rho)=\sqrt{\rho_{A}} \operatorname{Tr}_{B}\left(R R^{\dagger}\right) \sqrt{\rho_{A}},
$$

which implies that

$$
\operatorname{Tr}_{B}\left(R R^{\dagger}\right) \sqrt{\rho_{A}}=\sqrt{\rho_{A}}=\sqrt{\rho_{A}} \operatorname{Tr}_{B}\left(R R^{\dagger}\right) .
$$

Consequently,

$$
\rho_{A}^{2} \operatorname{Tr}_{B}\left(R R^{\dagger}\right)=\rho_{A}^{2}
$$

Since

$\rho^{2}=\sqrt{\rho} \rho \sqrt{\rho}=R^{\dagger}\left(\rho_{A} \otimes I_{B}\right) R R^{\dagger}\left(\rho_{A} \otimes I_{B}\right) R \leqslant R^{\dagger}\left(\rho_{A}^{2} \otimes I_{B}\right) R$,

one has

$$
\begin{aligned}
\operatorname{Tr}\left(\rho^{2}\right) & \leqslant \operatorname{Tr}\left(R^{\dagger}\left(\rho_{A}^{2} \otimes I_{B}\right) R\right) \\
& =\operatorname{Tr}\left(\left(\rho_{A}^{2} \otimes I_{B}\right) R R^{\dagger}\right) \\
& =\operatorname{Tr}_{A}\left(\operatorname{Tr}_{B}\left(\left(\rho_{A}^{2} \otimes I_{B}\right) R R^{\dagger}\right)\right) \\
& =\operatorname{Tr}_{A}\left(\rho_{A}^{2} \operatorname{Tr}_{B}\left(R R^{\dagger}\right)\right) \\
& =\operatorname{Tr}_{A}\left(\rho_{A}^{2}\right) \\
& =\operatorname{Tr}\left(\rho_{A}^{2}\right) .
\end{aligned}
$$

Similarly, one can check that $I_{A} \otimes \rho_{B}-\rho \geqslant 0$ implies that $\operatorname{Tr}\left(\rho_{B}^{2}\right) \geqslant \operatorname{Tr}\left(\rho^{2}\right)$. Therefore, the trace inequality criterion is weaker than the reduction criterion.

Remark. Although the criterion of Theorem 2.1 is weaker than the reduction criterion, it is easy to handle for some senses, since its form is simpler than the reduction criterion. The criterion of Theorem 2.1 is a complementarity for detecting entanglement of states.

This work was partially supported by the National Natural Science Foundation of China (11171249) and the Natural Science Foundation of Shanxi Province (2011021002-2). The authors also wish to give their thanks to the referees for their comments to improve the presentation of this paper.

1 Nielsen M A, Chuang I L. Quantum Computation and Quantum Information. Cambridge: Cambridge University Press, 2000

2 Horodecki R, Horodecki P, Horodecki M, et al. Quantum entanglement. Rev Mod Phys, 2009, 81: 865-942

3 Gühne O, Tóth G. Entanglement detection. Phys Rep, 2009, 474: 1-75

4 Peres A. Separability criterion for density matrices. Phys Rev Lett, 1996, 77: 1413-1418 
5 Zyczkowski K, Horodecki P, Sanpera A, et al. Volume of the set of separable states. Phys Rev A, 1998, 58: 883-896

6 Horodecki M, Horodecki P, Horodecki R. Separability of mixed states: Necessary and sufficient conditions. Phys Lett A, 1996, 223: $1-14$

7 Horodecki M, Horodecki P. Reduction criterion of separability and limits for a class of distillation protocols. Phys Rev A, 1999, 59: 42064216

8 Nielsen M A, Kempe J. Separable states are more disordered globally than locally. Phys Rev Lett, 2001, 86: 5184-5187

9 Horodecki P. Separability criterion and inseparable mixed states with positive partial transposition. Phys Lett A, 1997, 232: 333-339

10 Rudolph O. Further results on the cross norm criterion for separability. Quant Inf Proc, 2005, 4: 219-239

11 Chen $\mathrm{K}, \mathrm{Wu} \mathrm{L}$ A. A matrix realignment method for recognizing entanglement. Quant Inf Comput, 2003, 3: 193-202

12 Li M, Fei S M, Li-Jost X Q. Bell inequality, separability and entanglement distillation. Chin Sci Bull, 2011, 56: 945-954

13 Ren X Z, Cong H L, Wang X W, et al. Quantum entanglement of the binomial field interacting with a cascade three-level atom beyond the rotating wave approximation. Sci China Phys Mech Astron, 2011, 54 $1625-1630$
14 Di Y M, Liu S P, Liu D D. Entanglement for a two-parameter class of states in a high-dimension bipartite quantum system. Sci China Phys Mech Astron, 2010, 53: 1868-1872

15 Li X K, Li J L, Liu B, et al. The parametric symmetry and numbers of the entangled class of $2 \times M \times N$ system. Sci China Phys Mech Astron, 2010, 54: 1471-1475

16 Guo Y, Qi X F, Hou J C. Sufficient and necessary conditions of separability for bipartite pure states in infinite-dimensional systems. Chin Sci Bull, 2011, 56: 840-846

17 Hou J C, Qi X F. Constructing entanglement witness for infinitedimensional systems. Phys Rev A, 2010, 81: 062351

18 Wu S J, Anandan J. Some aspects of separability. Phys Lett A, 2002 , 297: 4-8

19 Rudolph O. Some aspects of separability revisited. Phys Lett A, 2004 321: 239-243

20 Werner R F. Quantum states with Einstein-Podolsky-Rosen correlations admitting a hidden-variable model. Phys Rev A, 1989, 40: 4277-4281

21 Holevo A S, Shirokov M E, Werner R F. Separability and entanglementbreaking in infinite dimensions. Russian Math Surveys, 2005, 60: 359_ 360

22 Douglas R G. On majorization, factorization, and range inclusion of operators an Hilbert space. Proc Amer Math Soc, 1966, 17: 413-415

Open Access This article is distributed under the terms of the Creative Commons Attribution License which permits any use, distribution, and reproduction in any medium, provided the original author(s) and source are credited. 